\title{
Lipidomics in vascular health: current perspectives
}

This article was published in the following Dove Press journal:

Vascular Health and Risk Management

12 June 2015

Number of times this article has been viewed

\section{Genovefa Kolovou' \\ Vana Kolovou ${ }^{1,2}$ \\ Sophie Mavrogeni' \\ 'Cardiology Department, ${ }^{2}$ Molecular Immunology Laboratory, Onassis \\ Cardiac Surgery Center, Athens, Greece}

Correspondence: Genovefa Kolovou Cardiology Department, Onassis Cardiac Surgery Center, 356 Sygrou Avenue, 176 74 Athens, Greece

Tel +30 2l 09493520

$\mathrm{Fax}+302109493336$

Email genovefa@kolovou.com
Abstract: Identifying the mechanisms that convert a healthy vascular wall to an atherosclerotic wall is of major importance since the consequences may lead to a shortened lifespan. Classical risk factors (age, smoking, obesity, diabetes mellitus, hypertension, and dyslipidemia) may result in the progression of atherosclerotic lesions by processes including inflammation and lipid accumulation. Thus, the evaluation of blood lipids and the full lipid complement produced by cells, organisms, or tissues (lipidomics) is an issue of importance. In this review, we shall describe the recent progress in vascular health research using lipidomic advances. We will begin with an overview of vascular wall biology and lipids, followed by a short analysis of lipidomics. Finally, we shall focus on the clinical implications of lipidomics and studies that have examined lipidomic approaches and vascular health.

Keywords: lipidomics, lipids, vascular, atherosclerosis, mass spectrometry

\section{Introduction}

Identifying the mechanisms that modify a healthy vascular wall to an atherosclerotic wall is of major importance, since the consequences (myocardial infarction, stroke, and others) may threaten human life..$^{1-4}$ Nowadays, it is well known that during atherogenesis, low-density lipoprotein (LDL) particles enter the arterial wall, either oxidized (ox) or enzymatically degraded, and aggregate. Afterwards, modified LDL particles follow the atherogenic pathway that leads to atherosclerotic plaque formation, instead of the pathway followed by native LDL particles, which supplies cholesterol to peripheral cells. Within the subendothelial space, the modified LDL particles are engaged by scavenger receptors located on macrophages and smooth muscle cells. Then, free cholesterol (FC) and cholesterol ester (CE) from the LDL particles accumulate within the lysosomes, ${ }^{5}$ where lysosomal acid lipase hydrolyzes CE to FC. FC, after leaving the lysosome, can be re-esterified and located in lipid droplets. In case of the increased accumulation of FC in the lysosome, the inhibition of lysosomal acid lipase activity is observed, leading to the progression of atherosclerosis. Native LDL particles are composed of approximately 3,000 lipid molecules. Lipids can be classified as hydrophobic or amphipathic molecules with carbanion- (fatty acids, polyketides, and others) or carbocation-based (prenols, sterols, and others) units. ${ }^{6}$ Lipids can also be divided into simple lipids (fatty acids, sterols, and others) or complex lipids (glycerophospholipids and glycosphingolipids), and they can be organized into categories that cover eukaryotic and prokaryotic sources.

Concerning lipidomics, the word entered PubMed in the year 2003. ${ }^{7}$ Since then, researchers have established a definition for lipidomics, which is derived from several 
fields with broad deviations. Generally, lipidomics is considered a part of metabolomics (genomics, transcriptomics, proteomics) and is described as the quantitative characterization of the full lipid complement produced by cells, organisms, or tissues. 8,9 The term "metabolomics" was first used by Fiehn, ${ }^{10}$ and was further developed by the Metabolomics Society http://www.metabolomicssociety.org/. Metabolomics is moving metabolic research, which is based on examining single pathways, to focus on complex metabolic networks. ${ }^{11}$ Metabolomics are closest to the phenotype of the subject (metabolites are the end product of the -omics pathway: genomics; transcriptomics; proteomics; and metabolomics) and allow they us to expand upon disease-causing mechanisms and link them with other -omics. Thus, the term "lipidomics" (the quantitative and molecular determination of lipid molecules) can be referred to as a research field that studies entire cellular lipidomes on a large scale. ${ }^{7}$ Cellular lipidomes, which were first reported in a journal in 2001, ${ }^{12}$ represent the whole pool of lipids within the cell. Thanks to recent advances in mass spectrometry (MS) technology, the evaluation of hundred of lipids that make up the lipidome in a single biological specimen is possible.

In this review, we will describe the recent progress in vascular health research using lipidomic advances. We shall begin with an overview of vascular wall biology and lipids, followed by a short analysis of lipidomics and the use of technology to evaluate lipidomics. Finally, we will focus on the clinical implications of lipidomics and studies that have examined lipidomic approaches and vascular health.

\section{Lipids and vascular wall biology}

The close relationship between plasma lipids (particularly CEs) and the vascular wall is well documented. ${ }^{13}$ Inside the vascular wall, $\mathrm{CE}$ accumulates in a shape of droplets in the cytosol or in lysosomes (as mentioned earlier). ${ }^{14}$ Normally, the infiltrating LDL particles, which cross the endothelium, contain a CE-rich core and polyunsaturated linoleic acid. ${ }^{15}$ The LDL particles enriched with monounsaturated cholesteryl oleate are usually bigger than the LDL particles enriched with polyunsaturated cholesteryl linoleate, and they bind to arterial proteoglycans more vigorously with the consequence of being trapped and forced to follow the atherosclerotic pathway leading to plaque formation. ${ }^{16}$

Of note is that lipids are able to bind to Toll-like receptors (TLRs) and can initiate intracellular signaling. The TLRs were found to have proatherogenic ${ }^{17}$ or protective actions. ${ }^{18}$ Seimon et $\mathrm{al}^{19}$ observed that ox phospholipids, oxLDL particles, and saturated fatty acids activate apoptosis in endoplasmic reticulum-stressed macrophages through a mechanism involving TLRs. Also, lipoprotein (a), a risk factor for cardiovascular disease that has been shown to be genetically linked to myocardial infarction, ${ }^{20}$ is activated in TLRs.

On the other hand, the removal of lipids from plaques into circulation can be performed by high-density lipoprotein (HDL) particles. ${ }^{21-24}$ Thus, studying the HDL lipid classes may give more information about the current status of atherosclerotic plaques (as will be discussed).

\section{Lipids}

Lipids are involved in many biological mechanisms by acting as membrane barriers; they are an energy source and are involved in signaling events, trafficking, and the sorting of macromolecules, which are the most important functions. ${ }^{25}$ The methods for lipid evaluation are usually focused on the assessment of total LDL and HDL cholesterol, as well as triglycerides. Although, these measurements still remain a fundamental part of everyday practice, lipidomic evaluation brought about the quantification of additional lipid molecular elements across varying classes (acylglycerols, sterols, sphingolipids, and others). ${ }^{7,8}$ Moreover, lipidomic evaluation permits a single, untargeted quantitative and qualitative snapshot of lipid concentrations within the whole cell, tissue, or body fluid being examined. Thus, whole-plasma lipidomics take a more global view of lipid metabolism and can provide a detailed picture of the abnormalities in lipid metabolism, which is in contrast to the studies of isolated lipoproteins. For example, Weir et $\mathrm{al}^{26}$ evaluated the lipid species (number $[\mathrm{n}]=312$ ) in obese subjects and found a correlation with sphingomyelin (SM), phosphatidylcholine (PC), phosphatidylethanolamine (PE), phosphatidylinositol, CEs, and triglycerides with obesity (1,076 participants of the San Antonio Family Heart Study [SAFHS]). Stübiger et al ${ }^{27}$ evaluated young subjects with familial hypercholesterolemia or familial combined hyperlipidemia and observed differences of SM/ $\mathrm{PC}$ and $\mathrm{PC} / \mathrm{lysoPC}$ ratios and positive correlations between SM versus LDL cholesterol and lysoPC versus very (V)LDL cholesterol between subjects with familial hypercholesterolemia and other study groups.

\section{Plaque composition}

Arterial wall dysfunction is caused by atherosclerosis, which consists of a slow lifetime variety of histological changes leading to plaque formation. ${ }^{4,28}$ The functional anatomy of plaques is dependent on the mechanisms by which lipids may provoke plaque instability and rupture. The intimal thickening 
is the first and clinically detectable manifestation of atherosclerosis in humans. The lesions present with foam and smooth muscle cells, and as they advance, with lipid pools located in the deeper intima in areas rich in proteoglycans, as well as with calcification. ${ }^{4}$ The broadest classification of atherosclerotic plaques includes stable and unstable plaques. Unstable plaques are characterized by high lipid load and, particularly, by $\mathrm{FC}$ and CEs, ${ }^{29}$ while stable plaques are characterized by phospholipids and triglyceride content. ${ }^{30}$ Stübiger et al, ${ }^{27}$ using the matrix-assisted laser desorption/ionization quadrupole ion trap time-of-flight (MALDI-QIT-TOF)-MS/ MS method, reported a positive correlation between oxPC levels and intima media thickness.

The major challenge in the evaluation of atherogenesis is the assessment of new risk factors when detecting the early formation of atherogenesis at a clinical level, in addition to the classic ones such as diet, exercise, LDL and VLDL cholesterol, and others. This is because the morphological tests (organ perfusion measurements or angiography) used for the diagnosis and classification of atherosclerosis are possible only in late-stage disease (they detect only established plaques) and they are not easily undertaken in large population studies. Recent advances in lipidomics make it possible to identify and quantify species from the plaque lipidome, and they have already revealed, that normal vessels in comparison with atherosclerosis-affected locations, have different lipid composition and particularly lysophospholipids and CEs. In the particular case of macrophages, higher levels of FC are related to foam cell formation. ${ }^{31}$ Furthermore, an increased $\mathrm{FC} / \mathrm{CE}$ ratio has been described in unstable atheromatous plaques in humans. ${ }^{32}$ Jové et a ${ }^{33}$ found that a high-fat diet provoked more changes in the aortic wall than in the plasma lipidome, and they suggested that vessels exhibit a "highfat molecular memory". Ox and enzymatically-modified (e)LDL particles play a crucial role in the early stages of atherogenesis. Their uptake by recruited macrophages leads to endolysosomal phospholipidosis (oxLDL) or foam cell formation (eLDL) (Figure 1). Hinterwirth et a ${ }^{34}$ used the antioxLDL antibodies conjugated to gold nanoparticles for the extraction and enrichment of oxPCs via the selective trapping of oxLDLs from plasma, combined with their detection by liquid chromatography (LC)-MS/MS, and found that both techniques can offer new possibilities for targeted lipidomics in lipoproteins, as well as for oxidative stress lipid biomarker screening.

Ramm Sander et $\mathrm{al}^{35}$ reported structural changes in LDL particles as a consequence of atherogenic modifications, as detected by nuclear magnetic resonance spectroscopy
(NMRS). Also, monitoring of the differential uptake of these LDL particles by macrophages could be followed. Particularly, eLDL-induced cytosolic lipid droplet formation could be detected. Moreover, eLDL-induced mobile lipids exhibited a greater proportion of polyunsaturated fatty acid chains (which correlated to apoptosis in vivo) when compared with lipids that were already present in macrophages prior to loading. This finding underlines the benefit of NMRS as a lipidomics tool to specifically monitor the mobile lipid pool, which is clearly different from the total lipid pool. Furthermore, the evaluation of isoprostanes by LC/MS methods offers the possibility to study the extent of oxidative stress in humans in various physiological and pathophysiological situations, such as atherosclerosis (Figure 1). ${ }^{36}$ Stegemann et al ${ }^{37}$ analyzed lipids in tissue sections and extracts from human endarterectomy specimens by shotgun lipidomics (in stable and unstable regions) from the same individual. Carotid plaque samples were enriched in CEs, PCs, lysoPCs, and SM species, as compared to radial arteries (control).

With respect to HDL particles, Camont et $\mathrm{al}^{38}$ revealed heterogeneity in the phosphosphingolipidome across human plasma HDL subpopulations using the LC-MS/MS approach, which paralleled the heterogeneity in key atheroprotective HDL functions. Furthermore, they observed that the HDL phosphosphingolipidome components were correlated with the cholesterol efflux capability of HDL from macrophages, antioxidative activity toward LDL, antithrombotic activity toward platelets, and antiapoptotic activity in endothelial cells. Thus, there is space for improvement of the classic lipid markers that are used in the estimation of future risk for major cardiovascular events caused by stable/unstable vascular disease.

\section{Lipidomics}

Lipidomics is still a relatively young discipline, which is rapidly developing and is considered to be a part of metabolomics. ${ }^{8}$ Lipidomics involves detailed documentation of particular cellular lipid species, including the type and number of atoms in each lipid species, as well as their stereoelectronic interactions with neighboring lipids and proteins. A number of methods have been introduced for the evaluation of the lipidome, such as "shotgun lipidomics" (which explores chemical and physical properties of each lipid class and involves direct infusion of the sample), ${ }^{39,40}$ targeted lipidomics (which obtains one or more of the lipid classes or subclasses, or molecular species of interest), ${ }^{41,42}$ and untargeted lipidomics (that identifies unknown lipid species). ${ }^{43}$ 


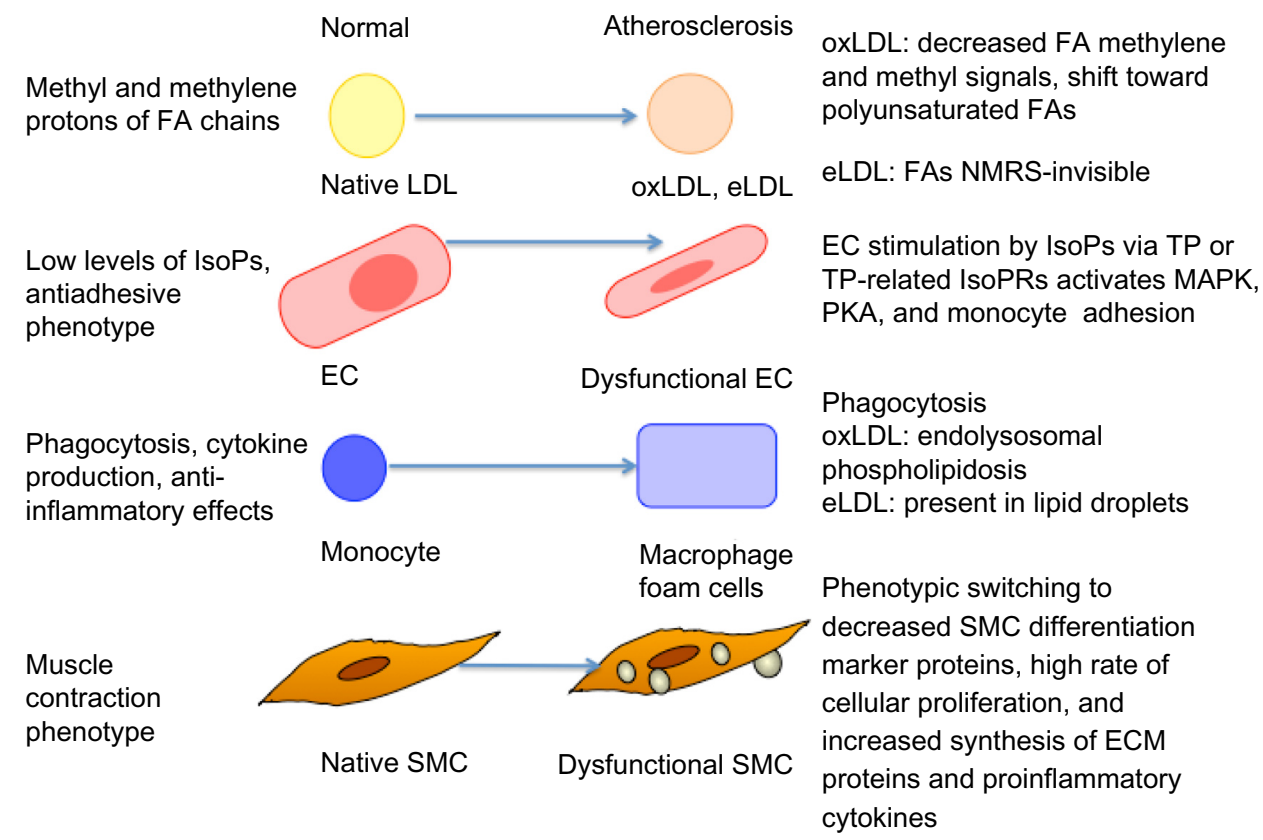

Figure I Atherosclerotic changes of various cells, as detected by NMRS or mass spectrometry/liquid chromatography methods.

Abbreviations: FA, fatty acid; oxLDL, oxidized low-density lipoprotein; LDL, low-density lipoprotein; eLDL, enzymatically-modified low-density lipoprotein; NMRS, nuclear magnetic resonance spectroscopy; IsoPs, isoprostanes; EC, endothelial cell; TP, thromboxane A2 receptor; IsoPRs, isoprostane receptors; MAPK, mitogen-activated protein kinase; PKA, protein kinase A; SMC, smooth muscle cell; ECM, extracellular matrix.

It was observed that every lipoprotein class was associated with a particular arrangement of lipids. For example, LDL particles are enriched with ceramide and SM, while HDL particles are enriched with PC, PE, and PE-based plasmalogens. The International Lipid Classification and Nomenclature Committee (ILCNC), the LIPID Metabolites and Pathways Strategy (LIPID MAPS Consortium), and the European Lipidomics Initiative (ELIfe) have classified the lipidomic nomenclature. ${ }^{44}$ This classification separates fatty acyls from other polyketides, glycerophospholipids from other glycerolipids, and sterol lipids from other prenols.

Current studies in lipidomics are focused on four main areas such as: 1) structural characterization of known and novel lipid species; 2) the development of methods for lipidomics analysis; 3 ) the evaluation of metabolic factors in a healthy and diseased settings; and 4) therapeutic response and side effects of drugs, which are analyzed in the "Lipidomics in clinical use" section.

\section{Structural characterization of known and novel lipid species}

In 1976, the International Union of Pure and Applied Chemistry and the International Union of Biochemistry and Molecular Biology (IUPAC-IUBMB) Commission on Biochemical Nomenclature ${ }^{45}$ published the lipid nomenclature. Since then, a number of novel lipid classes have been discovered. The
LIPID MAPS Consortium established a "Comprehensive Classification System for Lipids" based on well-defined chemical and biochemical principles, together with ontology, and classified lipids into eight categories (fatty acyls, glycerolipids, glycerophospholipids, sphingolipids, sterols, prenol lipids, saccharolipids, and polyketides) ${ }^{6}$

\section{Lipidomics - technology evaluation}

MS techniques and NMRS technology are the main techniques used for metabolomics evaluation (Table 1). MS techniques are largely used in the characterization of numerous lipid structures and their structure-specific functions. ${ }^{46}$ The use of MS techniques began in the 20th century and was indicated in organic, nuclear, geographical, and atomic chemistry, and only later was it applied to biological and medical research. Thus, the MS technique may examine certain areas of research, such as lipidomics, proteomics, metabolomics, gas-phase ion chemistry, proscribed chemicals, pharmacokinetics, protein/peptide chemistry, and others. The use of NMRS technology began circa 1970. NMRS technology, by measuring the magnetic spin of nuclei $\left({ }^{1} \mathrm{H},{ }^{13} \mathrm{C}\right.$, and $\left.{ }^{31} \mathrm{P}\right)$ contained in the study's metabolites, can identify structural and quantitative information of metabolites. ${ }^{11}$ Today, with NMRS technology, it is possible to identify up to 300 metabolites, ${ }^{46}$ and this is based on two molecular windows: lipoprotein lipid and low-molecular-mass/weight metabolite. ${ }^{47}$ 
Table I Various technologies and techniques at the forefront of lipidomics evaluation

\begin{tabular}{|c|c|}
\hline Name of the technique & Purpose of the technique \\
\hline \multicolumn{2}{|l|}{ MS } \\
\hline I. Tandem MS (MS/MS or $\left.\mathrm{MS}^{2}\right)$ & $\begin{array}{l}\text { Used in industry and research fields for drug discovery, diagnostics, and } \\
\text { bioanalyses. }{ }^{54,55}\end{array}$ \\
\hline $\begin{array}{l}\text { 2. Direct-infusion MS without prior chromatographic } \\
\text { separation (SL) } \\
\text { PIS } \\
\text { NL }\end{array}$ & $\begin{array}{l}\text { Exploits the chemical and physical properties of each lipid class to facilitate the } \\
\text { high-throughput analysis of a cellular lipidome directly from the organic extracts of } \\
\text { biological samples. }{ }^{56}\end{array}$ \\
\hline 3. MDMS-SL & $\begin{array}{l}\text { Covers the quantitative analysis of various classes of glycerophospholipids, } \\
\text { sphingolipids, and glycerolipids. }{ }^{56}\end{array}$ \\
\hline 4. Soft ionization techniques (ESI and MALDI) & $\begin{array}{l}\text { Analyzed carbohydrates, lipids, proteins, and nucleotides, as well as organic and } \\
\text { inorganic compounds. }{ }^{48,57}\end{array}$ \\
\hline LSI-MS & Mono- and polyhydroxylated fatty acids. ${ }^{49}$ \\
\hline ESI & $\begin{array}{l}\text { Identifies the major species of ceramide, SM, PC, lysoPC, PE, and its derivatives, } \\
\text { such as FC and CEs. }{ }^{48}\end{array}$ \\
\hline MALDI-QIT-TOF-MS/MS approach & Investigates atherogenic dyslipidemia in young patients with familial hyperlipidemia. ${ }^{27}$ \\
\hline MALDI-IMS & $\begin{array}{l}\text { Performs lipid analysis, avoiding extraction and/or separation steps, and displays the } \\
\text { in situ information. }{ }^{58,59}\end{array}$ \\
\hline 5. Atmospheric pressure chemical ionization & Principal enabling technology to tackle the lipidome..$^{56}$ \\
\hline \multicolumn{2}{|l|}{ Nuclear magnetic resonance spectroscopy } \\
\hline $\begin{array}{l}\text { Based on the measurement of the magnetic spin of nuclei } \\
\left({ }^{1} \mathrm{H},{ }^{13} \mathrm{C} \text {, and }{ }^{3 /} \mathrm{P}\right) \text { contained in the metabolites of interest }\end{array}$ & Lipid-protein interactions, identification of the structure of lipid membranes. ${ }^{35,60}$ \\
\hline \multicolumn{2}{|l|}{ Fluorescence spectroscopy } \\
\hline Fluorescence-activated cell sorting & Isolation of cells that show desirable characteristics by flow cytometry. ${ }^{61}$ \\
\hline Total internal reflection fluorescence microscopy & Explains membrane dynamics; shows adsorption of proteins and peptides to lipids. ${ }^{62}$ \\
\hline Fluorescence recovery after photobleaching & Measures the diffusion of molecules in a membrane. ${ }^{.2}$ \\
\hline Fluorescence correlation spectroscopy & General characterization of membranes..$^{62}$ \\
\hline \multicolumn{2}{|l|}{ Column chromatography } \\
\hline I. Chromatographic separation-coupled MS & Analyzes lipidomes. \\
\hline 2. Fast protein LC & Distinguishes serum lipoproteins. \\
\hline 3. LC-ESI-MS/MS & Distinguishes mono- and polyhydroxylated fatty acids. ${ }^{49}$ \\
\hline $\begin{array}{l}\text { 4. Reversed phase } \\
\text { Reversed-phase LC }\end{array}$ & $\begin{array}{l}\text { Lipid separation, based on the hydrophobic properties of lipids, the number of } \\
\text { carbons, and the degree of saturation. } \text {. }^{63,64}\end{array}$ \\
\hline 5. NP & Distinguishes lipid species according to their hydrophilic functionalities. \\
\hline NP-LC & Differs in the positioning of fatty acyls onto the glycerol backbone (sn-I, sn-2, sn-3); \\
\hline Silver ion NP-LC & can be found. ${ }^{64}$ \\
\hline Regioisomeric species & \\
\hline 6. Hydrophilic interaction LC & Distinguishes lipid species according to their hydrophilic functionalities. ${ }^{64}$ \\
\hline 7. Miniaturized column formats & Distinguishes ceramides in the lipidome. \\
\hline 8. Microfluidic chips & Identifies and quantifies the structure and function of lipids in biological systems. ${ }^{64}$ \\
\hline
\end{tabular}

Abbreviations: MS, mass spectrometry; SL, shotgun lipidomics; PIS, precursor ion scans; NL, neutral loss scans; MDMS-SL, multidimensional mass spectrometry-based shotgun lipidomics; ESI, electrospray ionization; MALDI, matrix-assisted laser desorption/ionization; LSI-MS, liquid secondary ion mass spectrometry; MALDI-QIT-TOF-MS/MS, matrix-assisted laser desorption/ionization quadrupole ion trap time-of-flight mass spectrometry; MALDI-IMS, matrix-assisted laser desorption/ionization imaging mass spectrometry; SM, sphingomyelin; PC, phosphatidylcholine; lysoPC, lysophosphatidylcholine; PE, phosphatidylethanolamine; FC, free cholesterol; CE, cholesterol ester; LC-ESI-MS/MS, liquid chromatography electrospray ionization tandem mass spectrometry; LC, liquid chromatography; NP, normal phase.

Fast protein LC technology has been introduced to distinct human serum lipoproteins. Electrospray ionization (ESI) techniques (soft ionization technology used to form either positive or negative ions without derivatization and decomposition) are performed to identify the major species of ceramide, SM, $\mathrm{PC}$, lysoPC, PE, and its derivatives, such as FC and CEs. ${ }^{48}$ LC tandem MS technology is a method that is used for the analysis of mono- and polyhydroxylated fatty acids. ${ }^{49}$ The analysis of mono- and polyhydroxylated fatty acids is helpful in establishing the role of lipid mediators in certain biological and/or pathological conditions. Moreover, other technical approaches for the identification of other lipid species are still being developed. For example, Blanksby and Mitchel ${ }^{50}$ have used ozone-induced dissociation to recognize the positions of double bonds in unsaturated fatty acids.

MS technology is still a developing method for evaluating lipidomics; however, one of the essential concerns of this method is the standardized data analysis. ${ }^{51}$ Lately, this has been improved..$^{52}$ Almeida et $\mathrm{al}^{53}$ have reported a novel shotgun lipidomics platform containing an Orbitrap Fusion 
MS equipped with an automated nanoelectrospray ion source. This analysis verified varied lipidome quantification covering more than 300 lipid species, 20 lipid classes, and more than 200 molecular glycerophospholipid species. Also, Stübiger et $\mathrm{al}^{27}$ introduced the MALDI-QIT-TOF-MS/MS and LC-ESISRM approaches to investigate atherogenic dyslipidemia in young patients with familial hyperlipidemia.

\section{Evaluation of metabolic factors in a healthy and diseased setting}

Lipid homeostasis is essential in health protection and its evaluation is fundamental. Lipidomics in a healthy setting is used to describe the specific lipids involved in dynamic physiological lipid changes, providing further insight into lipid control in cellular biology, such as the structural integrity of cellular membranes, signal transduction, and the regulation of membrane trafficking. On the other hand, any abnormalities in lipid metabolism play a significant role in many diseases, particularly in metabolic syndrome, diabetes mellitus, lipodystrophies, neurological disorders (Alzheimer's disease, Parkinson's disease, Niemann-Pick disease, multiple sclerosis, and others), central nervous system injury (stroke, traumatic brain injury, and spinal cord injury), infections, and others.

Thus, lipidomic investigation leading to the discovery of new biomarkers is crucial for preventing these diseases in their very early stages. For example, impaired mitochondrial function - particularly, fatty acyl compositions - were proposed as the potential cause of insulin resistance and/or diabetes progression. ${ }^{65}$ Also, by using lipidomic methodologies, the cell lipid composition of some infective pathogens is being progressively determined. ${ }^{66} \mathrm{~A}$ recent shotgun lipidomics study examined the effect of sulfatide supplementation on neuroblastoma cells. Lipidomic methodologies were also used to map lipid disorders in human muscles of a patient suffering from mulscular dystrophy. ${ }^{67}$

\section{Lipidomics in clinical use}

One of the main clinical uses of lipidomics is the monitoring of therapeutic responses and the potential side effects of existing drugs, as well as the evaluation of newly developed ones. In terms of lipid-lowering (LL) drugs such as statins (3-hydroxy-3-methylglutaryl-coenzyme A inhibitors), which are first-line LL drugs for the treatment of hypercholesterolemia, the evaluation of their efficacy was based on blood lipid concentration measurements. ${ }^{68}$ Lately, with the introduction of lipidomics, the LL effectiveness and side effects of statins were also extended to lipidomic evaluation. Laaksonen et al ${ }^{69}$ evaluated 132 plasma lipidomic analyses of subjects treated with simvastatin, atorvastatin, or placebo, and they found that the plasma lipidomic changes in the simvastatin group were associated with the muscle expression of the arachidonate 5-lipoxygenase-activating protein. They concluded that the plasma lipidomic profile might serve as a highly sensitive biomarker of statin-induced metabolic changes in muscle, and it might help clinicians to distinguish between subjects who should be treated with a lower statin dose to prevent possible toxicity. In the plasma lipidomics analysis of men with combined lipidemia, Chen et $\mathrm{al}^{70}$ found that the simvastatin treatment group exhibited a significant reduction in nine out of 33 species. Thus, significant reductions were observed in FC, CEs, triglycerides, PEs, and lysoPCs (which were the primary constituents in CEs), as well as in triglycerides in intermediate-density lipoprotein/ VLDL and LDL particles. ${ }^{45}$ Kaddurah-Daouk et al, ${ }^{71}$ using a targeted lipidomics platform, reported lipid modifications in blood samples of upper vs lower subgroup (defined by LDL cholesterol response to simvastatin LL effect). Particularly, baseline CEs and phospholipid metabolites were correlated with the LDL cholesterol response to LL treatment. C-reactive protein (CRP) response to therapy correlated with baseline plasmalogens, which are lipids involved in inflammation. Another group of LL drugs includes fibrates. Fibrates act as peroxisome proliferator-activated receptor $\alpha$ agonists. Yetukuri et $\mathrm{al}^{72}$ reported that fenofibrate induced HDL compositional changes that included increased apolipoprotein A-II and SM, as well as reduced lysoPC. The ethanolamine plasmalogens were reduced only in the subgroup of patients with elevated homocysteine levels.

Also, considerable progress was made according to newly developed drugs and lipidomics. Aerts et al ${ }^{73}$ developed an inhibitor of glucosylceramide synthase (an enzyme that catalyzes the conversion of ceramide to glycosphingolipids), which was administered to high-fat-fed or ob/ ob mice and Zucker diabetic fatty rats. The authors found lowered circulating glucose levels, improved oral glucose tolerance, improved insulin sensitivity in the muscle and liver, and a reduction of glycosphingolipid concentration in various tissues. Holland et $\mathrm{al}^{74}$ also found that the inhibition of ceramide synthesis improved glucose tolerance and prevented the onset of diabetes in obese rodents. Moreover, Zhao et $\mathrm{al}^{75}$ and Bijl et $\mathrm{al}^{76}$ found similar results in Zucker diabetic fatty rats and ob/ob rats, respectively. These findings indicate that modifications in sphingolipids can serve as theoretically applicable modulators of insulin action, and they show the new treatment approaches that have arisen due to lipidomics analysis. 


\section{Lipidomics and the vascular wall}

Plasma lipidomic biomarkers may identify global atherosclerotic risk or the condition of the vascular wall (see "Plaque composition"). ${ }^{77-80}$ Lipid classes are responsible for the main differentiations between a healthy and a diseased vascular wall. More than 300 lipid classes were already recognized in atherosclerotic plaques, particularly CEs, SMs, PC/lysoPC, and PCs. ${ }^{6,81}$ Significant modifications of CEs with linoleic acid and other polyunsaturated fatty acids were observed between healthy and diseased arteries. ${ }^{77}$ Thus, low levels of polyunsaturated fatty acids and high levels of linoleic acid in human atherosclerotic plaques expose them to inflammatory mediators ${ }^{78}$ and lead to the progression of atherosclerosis.

Meikle et a $\mathrm{l}^{43}$ evaluated plasma lipid profiles containing 305 lipids from 220 individuals (matched healthy controls, $\mathrm{n}=80$; those with stable angina, $\mathrm{n}=60$; and those with unstable coronary syndrome, $\mathrm{n}=80$ ) using ESI tandem MS technology. They found that ceramide, phosphatidylinositol, and PE species were positively associated with stable coronary artery disease, while lysoPC, ether-linked, and plasmalogen species of PC were negatively associated with stable disease (Figure 1).

The most desirable effect of antiatherosclerotic treatment is the regression of already-formed atherosclerotic plaques. In many statin treatment studies, a regression of atherosclerotic plaques has been reported. ${ }^{82-85}$ This clinical benefit of statin treatment is mainly correlated with the lowering of LDL cholesterol; however, other factors beyond LDL cholesterol lowering may be found. In two studies of statin treatment (the Study of Coronary Atheroma by InTravascular Ultrasound: Effect of Rosuvastatin Versus AtorvastatiN [SATURN] trial, ${ }^{83}$ and the Rosuvastatin and Atorvastatin in different Dosages and Reverse cholesterol transport [RADAR] trial ${ }^{86}$ ), prospective lipidomics analysis was evaluated. Atorvastatin and rosuvastatin decreased plasma SM concentrations. Rosuvastatin increased the plasma concentration of PCs, while atorvastatin reduced the plasma concentrations of PCs. The higher reduction in the SM/ $\mathrm{SM}+\mathrm{PC}$ ratio was observed with rosuvastatin treatment when compared with atorvastatin. ${ }^{86}$ It is noteworthy to mention that nearly five decades ago, $\mathrm{Smith}^{87}$ found the accumulation of SM in human atheromatous plaques, while Schissel et $\mathrm{l}^{88}$ found that the ratio of $\mathrm{SM} / \mathrm{SM}+\mathrm{PC}$ correlates with atherogenesis. Also, Noël et a ${ }^{89}$ observed the elevation of SM levels in subjects with familial hypercholesterolemia, a disease that frequently leads to premature cardiovascular disease.

Improvement in the SM/PC ratio by statins may suggest the reduced susceptibility of SM to hydrolysis by sphingomyelinase within the vessel wall. ${ }^{88,90}$ It worth mentioning that the inhibition of SM synthesis has been shown to reduce plasma total cholesterol and triglyceride concentration levels, and to increase HDL cholesterol concentrations in apolipoprotein E-knockout (apoE-/-) mice, which is a useful animal model for experimental atherosclerosis research with inactivated gene coding for the apolipoprotein E protein. ${ }^{91}$ These lipid changes were associated with increased plaque regression..$^{92,93}$

However, more studies have to be designed to determine the correlations between lipidomics and atherosclerosis. Voros et $\mathrm{a}^{94}$ have designed a prospective multicenter study (Genetic Loci and the Burden of Atherosclerotic Lesions) that will examine the biological associations between genomic, proteomic, metabolomic, lipidomic, and phenotypic factors of atherosclerosis from a large $(7,500)$ number of biological factors. Patients will undergo noncontrast-enhanced coronary calcium scanning by computed tomography (CT), coronary artery CT angiography, whole-genome sequencing, DNA methylation, whole blood-based transcriptome sequencing, unbiased proteomics based on MS, metabolomics, and lipidomics. ${ }^{37}$ Thus, the conclusions concerning panomics and atherosclerosis can be safely drawn.

\section{Conclusion}

Lipidomics can provide an unbiased field for the investigation of lipids within atherosclerosis. The introduction of lipidomics analysis will allow us to develop patterns that could identify cardiovascular risk factors beyond classical plasma lipids. Additionally, the combinations of lipidomic analysis and classical risk factors may lead us to form better judgments on whom to treat. Moreover, lipidomics analysis can lead us to new therapeutic targets and to novel therapeutic agents. Also, the side effects of the drugs may be better understood and prevented. Thus, lipidomics is a discipline that is rapidly developing and can offer several new strategies.

\section{Disclosure}

The authors report no conflicts of interest in this work.

\section{References}

1. Kolovou GD, Kolovou V, Mavrogeni S. We are ageing. Biomed Res Int. 2014;2014:808307.

2. Vasiliadis I, Kolovou G, Mavrogeni S, Nair DR, Mikhailidis DP. Sudden cardiac death and diabetes mellitus. J Diabetes Complications. 2014;28(4):573-579.

3. Avery P, Barzilai N, Benetos A, et al. Ageing, longevity, exceptional longevity and related genetic and non genetics markers: panel statement. Curr Vasc Pharmacol. 2014;12(5):659-661.

4. Silvestre-Roig C, de Winther MP, Weber C, Daemen MJ, Lutgens E, Soehnlein O. Atherosclerotic plaque destabilization: mechanisms, models, and therapeutic strategies. Circ Res. 2014;114(1):214-226. 
5. Jerome WG, Yancey PG. The role of microscopy in understanding atherosclerotic lysosomal lipid metabolism. Microsc Microanal. 2003;9(1): 54-67.

6. Fahy E, Subramaniam S, Brown HA, et al. A comprehensive classification system for lipids. J Lipid Res. 2005;46(5):839-861.

7. Han X, Gross RW. Global analyses of cellular lipidomes directly from crude extracts of biological samples by ESI mass spectrometry: a bridge to lipidomics. J Lipid Res. 2003;44(6):1071-1079.

8. Wenk MR. The emerging field of lipidomics. Nat Rev Drug Discov. 2005;4(7):594-610.

9. Dennis EA. Lipidomics joins the omics evolution. Proc Natl Acad Sci US A. 2009;106(7):2089-2090.

10. Fiehn O. Combining genomics, metabolome analysis, and biochemical modelling to understand metabolic networks. Comp Funct Genomics. 2001;2(3):155-168.

11. Griffin JL, Atherton H, Shockcor J, Atzori L. Metabolomics as a tool for cardiac research. Nat Rev Cardiol. 2011;8(11):630-643.

12. Kishimoto K, Urade R, Ogawa T, Moriyama T. Nondestructive quantification of neutral lipids by thin-layer chromatography and laser-fluorescent scanning: suitable methods for "lipidome" analysis. Biochem Biophys Res Commun. 2001;281(3):657-662.

13. Brown AJ, Leong SL, Dean RT, Jessup W. 7-Hydroperoxycholesterol and its products in oxidized low density lipoprotein and human atherosclerotic plaque. J Lipid Res. 1997;38(9):1730-1745.

14. Shio H, Haley NJ, Fowler S. Characterization of lipid-laden aortic cells from cholesterol-fed rabbits. III. Intracellular localization of cholesterol and cholesteryl ester. Lab Invest. 1979;41(2):160-167.

15. Mallat Z, Nakamura T, Ohan J, et al. The relationship of hydroxyeicosatetraenoic acids and F2-isoprostanes to plaque instability in human carotid atherosclerosis. J Clin Invest. 1999;103(3):421-427.

16. Degirolamo C, Shelness GS, Rudel LL. LDL cholesteryl oleate as a predictor for atherosclerosis: evidence from human and animal studies on dietary fat. J Lipid Res. 2009;50 Suppl:S434-S439.

17. Monaco C, Gregan SM, Navin TJ, Foxwell BM, Davies AH, Feldmann M. Toll-like receptor-2 mediates inflammation and matrix degradation in human atherosclerosis. Circulation. 2009;120(24):2462-2469.

18. Cole JE, Navin TJ, Cross AJ, et al. Unexpected protective role for Tolllike receptor 3 in the arterial wall. Proc Natl Acad Sci U S A. 2011; 108(6):2372-2377.

19. Seimon TA, Nadolski MJ, Liao X, et al. Atherogenic lipids and lipoproteins trigger CD36-TLR2-dependent apoptosis in macrophages undergoing endoplasmic reticulum stress. Cell Metab. 2010;12(5): 467-482.

20. Holmer SR, Hengstenberg C, Kraft HG, et al. Association of polymorphisms of the apolipoprotein(a) gene with lipoprotein(a) levels and myocardial infarction. Circulation. 2003;107(5):696-701.

21. Meikle PJ, Wong G, Barlow CK, Kingwell BA. Lipidomics: potential role in risk prediction and therapeutic monitoring for diabetes and cardiovascular disease. Pharmacol Ther. 2014;143(1):12-23.

22. Kontush A, Chantepie S, Chapman MJ. Small, dense HDL particles exert potent protection of atherogenic LDL against oxidative stress. Arterioscler Thromb Vasc Biol. 2003;23(10):1881-1888.

23. Zerrad-Saadi A, Therond P, Chantepie S, et al. HDL3-mediated inactivation of LDL-associated phospholipid hydroperoxides is determined by the redox status of apolipoprotein A-I and HDL particle surface lipid rigidity: relevance to inflammation and atherogenesis. Arterioscler Thromb Vasc Biol. 2009;29(12):2169-2175.

24. Kolovou GD, Cokkinos DV. Low serum levels of high-density lipoprotein cholesterol and hypolipidaemic treatment. Curr Med Res Opin. 2002;18(5):265-268.

25. Phillips MC. Molecular mechanisms of cellular cholesterol efflux. J Biol Chem. 2014;289(35):24020-24029.

26. Weir JM, Wong G, Barlow CK, et al. Plasma lipid profiling in a large population-based cohort. J Lipid Res. 2013;54(10):2898-2908.

27. Stübiger G, Aldover-Macasaet E, Bicker W, et al. Targeted profiling of atherogenic phospholipids in human plasma and lipoproteins of hyperlipidemic patients using MALDI-QIT-TOF-MS/MS. Atherosclerosis. 2012;224(1):177-186.
28. Halvorsen B, Otterdal K, Dahl TB, et al. Atherosclerotic plaque stability - what determines the fate of a plaque? Prog Cardiovasc Dis. 2008;51(3):183-194.

29. Davies MJ. Stability and instability: two faces of coronary atherosclerosis. The Paul Dudley White Lecture 1995. Circulation. 1996;94(8):2013-2020.

30. Felton CV, Crook D, Davies MJ, Oliver MF. Relation of plaque lipid composition and morphology to the stability of human aortic plaques. Arterioscler Thromb Vasc Biol. 1997;17(7):1337-1345.

31. Tabas I. Consequences of cellular cholesterol accumulation: basic concepts and physiological implications. J Clin Invest. 2002;110(7): 905-911.

32. Chen Z, Ichetovkin M, Kurtz M, et al. Cholesterol in human atherosclerotic plaque is a marker for underlying disease state and plaque vulnerability. Lipids Health Dis. 2010;9:61

33. Jové M, Ayala V, Ramírez-Núñez O, et al. Lipidomic and metabolomic analyses reveal potential plasma biomarkers of early atheromatous plaque formation in hamsters. Cardiovasc Res. 2013;97(4):642-652.

34. Hinterwirth H, Stübiger G, Lindner W, Lämmerhofer M. Gold nanoparticle-conjugated anti-oxidized low-density lipoprotein antibodies for targeted lipidomics of oxidative stress biomarkers. Anal Chem. 2013;85(17):8376-8384.

35. Ramm Sander P, Peer M, Grandl M, Bogdahn U, Schmitz G, Kalbitzer HR. NMR spectroscopy of macrophages loaded with native, oxidized or enzymatically degraded lipoproteins. PLoS One. 2013;8(2):e56360.

36. Minuz P, Fava C, Lechi A. Lipid peroxidation, isoprostanes and vascular damage. Pharmacol Rep. 2006;58 Suppl:57-68.

37. Stegemann C, Drozdov I, Shalhoub J, et al. Comparative lipidomics profiling of human atherosclerotic plaques. Circ Cardiovasc Genet. 2011;4(3):232-242.

38. Camont L, Lhomme M, Rached F, et al. Small, dense high-density lipoprotein-3 particles are enriched in negatively charged phospholipids: relevance to cellular cholesterol efflux, antioxidative, antithrombotic, anti-inflammatory, and antiapoptotic functionalities. Arterioscler Thromb Vasc Biol. 2013;33(12):2715-2723.

39. Yang K, Cheng H, Gross RW, Han X. Automated lipid identification and quantification by multidimensional mass spectrometry-based shotgun lipidomics. Anal Chem. 2009;81(11):4356-4368.

40. Han X, Gross RW. Shotgun lipidomics: electrospray ionization mass spectrometric analysis and quantitation of cellular lipidomes directly from crude extracts of biological samples. Mass Spectrom Rev. 2005;24(3):367-412.

41. Quehenberger O, Armando AM, Brown AH, et al. Lipidomics reveals a remarkable diversity of lipids in human plasma. J Lipid Res. 2010; 51(11):3299-3305.

42. Dennis EA, Deems RA, Harkewicz R, et al. A mouse macrophage lipidome. J Biol Chem. 2010;285(51):39976-39985.

43. Meikle PJ, Wong G, Tsorotes D, et al. Plasma lipidomic analysis of stable and unstable coronary artery disease. Arterioscler Thromb Vasc Biol. 2011;31(11):2723-2732.

44. Wenk MR. Lipidomics: new tools and applications. Cell. 2010;143(6): 888-895.

45. The nomenclature of lipids. Recommendations (1976) IUPAC-IUB Commission on Biochemical Nomenclature. Lipids. 1977;12(6):455-468.

46. Rasmiena AA, Ng TW, Meikle PJ. Metabolomics and ischaemic heart disease. Clin Sci (Lond). 2013;124(5):289-306.

47. Soininen $\mathrm{P}$, Kangas AJ, Würtz $\mathrm{P}$, et al. High-throughput serum NMR metabonomics for cost-effective holistic studies on systemic metabolism. Analyst. 2009;134(9):1781-1785.

48. Wiesner P, Leidl K, Boettcher A, Schmitz G, Liebisch G. Lipid profiling of FPLC-separated lipoprotein fractions by electrospray ionization tandem mass spectrometry. J Lipid Res. 2009;50(3):574-585.

49. Isobe Y, Arita M, Matsueda S, et al. Identification and structure determination of novel anti-inflammatory mediator resolvin E3, 17,18-dihydroxyeicosapentaenoic acid. J Biol Chem. 2012;287(13): 10525-10534.

50. Blanksby SJ, Mitchell TW. Advances in mass spectrometry for lipidomics. Annu Rev Anal Chem (Palo Alto Calif). 2010;3: $433-465$ 
51. Ejsing CS, Duchoslav E, Sampaio J, et al. Automated identification and quantification of glycerophospholipid molecular species by multiple precursor ion scanning. Anal Chem. 2006;78(17):6202-6214.

52. Ejsing CS, Sampaio JL, Surendranath V, et al. Global analysis of the yeast lipidome by quantitative shotgun mass spectrometry. Proc Natl Acad Sci U S A. 2009;106(7):2136-2141.

53. Almeida R, Pauling JK, Sokol E, Hannibal-Bach HK, Ejsing CS. Comprehensive lipidome analysis by shotgun lipidomics on a hybrid quadrupole-orbitrap-linear ion trap mass spectrometer. J Am Soc Mass Spectrom. 2015;26(1):133-148.

54. Di Girolamo F, Lante I, Muraca M, Putignani L. The role of mass spectrometry in the "omics" era. Curr Org Chem. 2013;17(23):2891-2905.

55. Feng X, Liu X, Luo Q, Liu BF. Mass spectrometry in systems biology: an overview. Mass Spectrom Rev. 2008;27(6):635-660.

56. Han X, Yang K, Gross RW. Multi-dimensional mass spectrometry-based shotgun lipidomics and novel strategies for lipidomic analyses. Mass Spectrom Rev. 2012;31(1):134-178.

57. Fenn JB, Mann M, Meng CK, Wong SF, Whitehouse CM. Electrospray ionization for mass spectrometry of large biomolecules. Science. 1989;246(4926):64-71.

58. Griffiths RL, Sarsby J, Guggenheim EJ, et al. Formal lithium fixation improves direct analysis of lipids in tissue by mass spectrometry. Anal Chem. 2013;85(15):7146-7153.

59. Longuespée R, Boyon C, Desmons A, etal. Spectroimmunohistochemistry: a novel form of MALDI mass spectrometry imaging coupled to immunohistochemistry for tracking antibodies. OMICS. 2014;18(2): 132-141.

60. Gawrisch K, Eldho NV, Polozov IV. Novel NMR tools to study structure and dynamics of biomembranes. Chem Phys Lipids. 2002;116(1-2): 135-151.

61. Horn PJ, Chapman KD. Lipidomics in situ: insights into plant lipid metabolism from high resolution spatial maps of metabolites. Prog Lipid Res. 2014;54:32-52.

62. Sezgin E, Schwille P. Fluorescence techniques to study lipid dynamics. Cold Spring Harb Perspect Biol. 2011;3(11):a009803.

63. Gao X, Zhang Q, Meng D, et al. A reversed-phase capillary ultraperformance liquid chromatography-mass spectrometry (UPLC-MS) method for comprehensive top-down/bottom-up lipid profiling. Anal Bioanal Chem. 2012;402(9):2923-2933

64. Sandra K, Sandra P. Lipidomics from an analytical perspective. Curr Opin Chem Biol. 2013;17(5):847-853.

65. Li J, Romestaing C, Han X, et al. Cardiolipin remodeling by ALCAT1 links oxidative stress and mitochondrial dysfunction to obesity. Cell Metab. 2010;12(2):154-165.

66. Chan R, Uchil PD, Jin J, et al. Retroviruses human immunodeficiency virus and murine leukemia virus are enriched in phosphoinositides. J Virol. 2008;82(22):11228-11238.

67. Brunelle A, Laprévote O. Lipid imaging with cluster time-of-flight secondary ion mass spectrometry. Anal Bioanal Chem. 2009;393(1): 31-35.

68. Kolovou GD, Katerina A, Ioannis V, Cokkinos DV. Simvastatin: two decades in a circle. Cardiovasc Ther. 2008;26(2):166-178.

69. Laaksonen R, Jänis MT, Oresic M. Lipidomics-based safety biomarkers for lipid-lowering treatments. Angiology. 2008;59(2 Suppl): $65 \mathrm{~S}-68 \mathrm{~S}$.

70. Chen F, Maridakis V, O’Neill EA, et al. The effects of simvastatin treatment on plasma lipid-related biomarkers in men with dyslipidaemia. Biomarkers. 2011;16(4):321-333.

71. Kaddurah-Daouk R, Baillie RA, Zhu H, et al. Lipidomic analysis of variation in response to simvastatin in the Cholesterol and Pharmacogenetics Study. Metabolomics. 2010;6(2):191-201.

72. Yetukuri L, Huopaniemi I, Koivuniemi A, et al. High density lipoprotein structural changes and drug response in lipidomic profiles following the long-term fenofibrate therapy in the FIELD substudy. PLoS One. 2011;6(8):e23589.

73. Aerts JM, Ottenhoff R, Powlson AS, et al. Pharmacological inhibition of glucosylceramide synthase enhances insulin sensitivity. Diabetes. 2007;56(5):1341-1349.
74. Holland WL, Brozinick JT, Wang LP, et al. Inhibition of ceramide synthesis ameliorates glucocorticoid-, saturated-fat-, and obesityinduced insulin resistance. Cell Metab. 2007;5(3):167-179.

75. Zhao H, Przybylska M, Wu IH, et al. Inhibiting glycosphingolipid synthesis improves glycemic control and insulin sensitivity in animal models of type 2 diabetes. Diabetes. 2007;56(5):1210-1218.

76. Bij1 N, Sokolović M, Vrins C, et al. Modulation of glycosphingolipid metabolism significantly improves hepatic insulin sensitivity and reverses hepatic steatosis in mice. Hepatology. 2009;50(5):1431-1441.

77. Didangelos A, Stegemann C, Mayr M. The -omics era: proteomics and lipidomics in vascular research. Atherosclerosis. 2012;221(1):12-17.

78. Ekroos K, Jänis M, Tarasov K, Hurme R, Laaksonen R. Lipidomics: a tool for studies of atherosclerosis. Curr Atheroscler Rep. 2010;12(4): 273-281.

79. Arita M. Mediator lipidomics in acute inflammation and resolution. J Biochem. 2012;152(4):313-319.

80. Lam SM, Shui G. Lipidomics as a principal tool for advancing biomedical research. J Genet Genomics. 2013;40(8):375-390.

81. Bou Khalil M, Hou W, Zhou H, et al. Lipidomics era: accomplishments and challenges. Mass Spectrom Rev. 2010;29(6):877-929.

82. Feig JE, Feig JL, Kini AS. Statins, atherosclerosis regression and HDL: Insights from within the plaque. Int J Cardiol. 2015;189:168-171.

83. Nicholls SJ, Borgman M, Nissen SE, et al. Impact of statins on progression of atherosclerosis: rationale and design of SATURN (Study of Coronary Atheroma by InTravascular Ultrasound: effect of Rosuvastatin versus AtorvastatiN). Curr Med Res Opin. 2011;27(6):1119-1129.

84. Puri R, Libby P, Nissen SE, et al. Long-term effects of maximally intensive statin therapy on changes in coronary atheroma composition: insights from SATURN. Eur Heart J Cardiovasc Imaging. 2014;15(4): 380-388.

85. Albert MA, Glynn RJ, Fonseca FA, et al. Race, ethnicity, and the efficacy of rosuvastatin in primary prevention: the Justification for the Use of Statins in Prevention: an Intervention Trial Evaluating Rosuvastatin (JUPITER) trial. Am Heart J. 2011;162(1):106-114. e2.

86. Bergheanu SC, Reijmers T, Zwinderman AH, et al. Lipidomic approach to evaluate rosuvastatin and atorvastatin at various dosages: investigating differential effects among statins. Curr Med Res Opin. 2008;24(9):2477-2487.

87. Smith EB. Intimal and medial lipids in human aortas. Lancet. 1960; 1(7128):799-803.

88. Schissel SL, Jiang X, Tweedie-Hardman J, et al. Secretory sphingomyelinase, a product of the acid sphingomyelinase gene, can hydrolyze atherogenic lipoproteins at neutral $\mathrm{pH}$. Implications for atherosclerotic lesion development. J Biol Chem. 1998;273(5):2738-2746.

89. Noël C, Marcel YL, Davignon J. Plasma phospholipids in the different types of primary hyperlipoproteinemia. J Lab Clin Med. 1972;79(4): 611-621.

90. Jeong Ts, Schissel SL, Tabas I, Pownall HJ, Tall AR, Jiang X. Increased sphingomyelin content of plasma lipoproteins in apolipoprotein E knockout mice reflects combined production and catabolic defects and enhances reactivity with mammalian sphingomyelinase. J Clin Invest. 1998;101(4):905-912.

91. Kolovou G, Anagnostopoulou K, Mikhailidis DP, Cokkinos DV. Apolipoprotein E knockout models. Curr Pharm Des. 2008;14(4) 338-351.

92. Park TS, Panek RL, Rekhter MD, et al. Modulation of lipoprotein metabolism by inhibition of sphingomyelin synthesis in ApoE knockout mice. Atherosclerosis. 2006;189(2):264-272.

93. Oestvang J, Bonnefont-Rousselot D, Ninio E, Hakala JK, Johansen B, Anthonsen MW. Modification of LDL with human secretory phospholipase A(2) or sphingomyelinase promotes its arachidonic acid-releasing propensity. J Lipid Res. 2004;45(5):831-838.

94. Voros S, Maurovich-Horvat P, Marvasty IB, et al. Precision phenotyping, panomics, and system-level bioinformatics to delineate complex biologies of atherosclerosis: rationale and design of the "Genetic Loci and the Burden of Atherosclerotic Lesions" study. J Cardiovasc Comput Tomogr. 2014;8(6):442-451. 


\section{Publish your work in this journal}

Vascular Health and Risk Management is an international, peerreviewed journal of therapeutics and risk management, focusing on concise rapid reporting of clinical studies on the processes involved in the maintenance of vascular health; the monitoring, prevention and treatment of vascular disease and its sequelae; and the involvement of

metabolic disorders, particularly diabetes. This journal is indexed on PubMed Central and MedLine. The manuscript management system is completely online and includes a very quick and fair peer-review system, which is all easy to use. Visit http://www.dovepress.com/ testimonials.php to read real quotes from published authors.

Submit your manuscript here: http://www.dovepress.com/vascular-health-and-risk-management-journal 